\section{SOVIET QUANTUM RESEARCH}

\section{Methods of Quantum Chemistry}

By M. G. Veselov. Translated by Scripta Technica, Inc. Translation edited by S. Chomet. Pp. xi+181. (New York : Academic Press lnc.; London: Academic Press Inc. (London), Ltd., 1965.) \$7.50.

\section{Advances in Quantum Chemistry}

Vol. 2. Edited by Per-Olov Löwdin. Pp. xi +372. (New York : Academic Press, Inc. ; London: Academic Press, Inc. (London), Ltd., 1965.) $116 s$.

THE current research literature of the U.S.S.R. can nowadays be read translated into English; text-books, research monographs, and even review periodicals cannot. A few books recognized as outstanding, like the works of Landau and Lifschitz, have appeared in translation several years after first publication, but most are never seen at all in western countries, and one has little idea of the character and scalo of such secondary publishing in comparison with the bewildering abundance in the West. Do scientists in the U.S.S.R., in reflective assessments of recent work, assign importance to the same discoveries? Do they have distinctive scientific attitudes to the role of theory in chemistry? Is there a distinguishable official "line" informing their more critical writings and tending to influence the course of original work? On presont-day evidence, supported by the evidence of Methods of Quantum Chemistry, there are no such differences. Indeed, there seems no particular reason why this book, which is a collection of reviews, should have been singled out for translation because most of the material, while perfectly soundly presented, is widely available in English language review publications. This is true of "Semi-empirical Methods in the Theory of Small Molecules" (T. K. Rebane), of the chapters on molecular orbital methods (Mestechkin), and theoretical reactivity studies in conjugated systems (Tupitsyn and Adamov). Nikitin's article on "Interelectron and NonAdiabatic Interaction in Long Molecules", and to a lesser extent Rebane's on finite difference methods in molecular orbital theory, havo greater interest on account of their discussions of the application of less familiar theoretical methods. M. G. Veselov's connexion with the book is that of editor, a fact which is not disclosed on the cover and title page. It is regrettable that he is not also one of the authors.

The second Advances in Quantum Chemistry is well up to the standard of the first. The articles are by Boys and Rajagopal, Fischer-Hjalmars, Blinder, McWeeny and Steiner, Jansen, Jehle and Löwdin. Good reviews are always to bo welcomed; and as the original literature slips out of reach by its sheer quantity, recovery in digests and comprehensive reviews as good as in these Advances is the more worth while and essential.

\section{P. Craig}

\section{ONE-DIMENSIONAL FLAMES}

\section{Flame Structure}

By R. M. Fristrom and A. A. Westenberg. (McGraw-Hill Series in Advanced Chemistry.) Pp. xiii +424 . (New York : MoGraw-Hill Book Company, Inc.; Maidenhead : MeGraw-Hill Publishing Company, Ltd., 1965.) $\$ 17.50 ; 140 s$.

A FLAME is, at first sight, the obvious medium for the experimental investigation of high temperature processes. It may be time-invariant phenomenon, almost completely free from surface effects and cheap to set up. Flames can be produced with a wide range of temperature and composition. However, the disadvantages of flameshaving many components and large temperature and con- centration gradients in the reaction zone-have caused many research workers interested in physical and chemical processes at high temperatures to dismiss flames as too complex to be suitable media for research. The authors of Flame Structure believe that this view is too pessimistic and their belief is fully substantiated by this book. There is no denying, of course, that flames are more complex than some other experimental media and that the steep gradients which exist in them make the analysis of flame data to obtain chemical kinetic information more difficult. These gradients can, however, be measured accurately and taken into account in many cases and the expenditure of effort involved is amply repaid by the results obtained.

This book is intended for combustion research workers, in particular for those working in the field of gas phase reaction kinetics. It is concerned primarily with measurements of the properties of pre-mixed, laminar flames and with the interpretation of these measurements to obtain information on fundamental physical and chemical processes in flames. This type of flame is chosen because it approximates closely to a one-dimensional flow system and is therefore the most amenable to quantitative analysis. It is also the best type of flame to use to assess the validity and accuracy of some of the experimental techniques commonly used in combustion studies.

The book begins by dealing with the basic equations needed in discussing flame processes, together with an essential amount of relevant information on transport processes, thermodynamies and chemical kinetics. The basic equations for the conservation of energy and the conservation of species are given with emphasis on the forms of the equations applicablo to flat and spherically symmetrical flames. The information which can be obtained from these equations is discussed as are the flame variables which have to be measured to do so.

The second, and principal, section deals with the practical measurement of the properties of one-dimensional flames. It begins with a description of different types of burners and of the ancillary flow metering and pumping equipment. This is followed by accounts of experimental techniques used to obtain flame velocities, temperature profiles, the concentration profiles of stable species (by probe sampling), the concentration profiles of free radicals and ions and by accounts of techniques for measuring distances and positions in flames, and obtaining flame photographs.

Throughout this section careful attention is paid to essential practical details and clarity of presentation. Each technique is illustrated by numerous excellent diagrams-somewhat over-elaborate in some cases-and photographs. In addition there are sevoral compact summaries of useful practical data, numerous references and a critical evaluation of the accuracy of the results obtained by different methods of measuring the same physical quantity. Most of these techniques are not, of course, limited in their usefulness to one-dimensional flames but can be used in many combustion systems. Because of this, Flame Structure will also appeal to research workers who study flames other than the idealized ones with which the book is mainly concerned. The weakest parts are those dealing with spectroscopic measurements of flames. Descriptions of spectroseopic methods of determining flame temperatures and species concentrations are not given at the same depth and with the same amount of detail as are descriptions of the other tech niques.

The chapter summarizing values obtained experimentally for the diffusion coefficients and thermal conductivities of binary mixtures of gases is particularly valuable. Included also are the means for calculating the values of these transport quantities at flame temperatures either from the kinetic theory of gases or from values which have been obtained at lower temperatures. As the authors say, "The magnitude of the diffusion velocities 